\title{
A Barocycler-based Concurrent Multi-Omics Method to Assess Molecular Changes Associated with Atherosclerosis using Small Amounts of Arterial Tissue from a Single Mouse
}

Jihan Talib ${ }^{1,2 \#}$, Peter G. Hains ${ }^{3 \#}$, Sergey Tumanov ${ }^{1 \#}$, Mark P. Hodson ${ }^{4,5}$, Phillip J. Robinson ${ }^{3}$, Roland Stocker ${ }^{1,2 *}$

${ }^{1}$ Vascular Biology Division, Victor Chang Cardiac Research Institute, Lowy Packer Building, 405 Liverpool Street, Darlinghurst NSW 2010, Australia; 2St Vincent's Clinical School, University of New South Wales Medicine, NSW 2050, Australia; ${ }^{3}$ Cell Signalling Unit, Children's Medical Research Institute, The University of Sydney, 214 Hawkesbury Rd, Westmead NSW 2145, Australia; ${ }^{4}$ Freedman Foundation Metabolomics Facility, Victor Chang Innovation Centre, Victor Chang Cardiac Research Institute, Lowy Packer Building, 405 Liverpool Street, Darlinghurst NSW 2010, Australia; ${ }^{5}$ School of Pharmacy, University of Queensland, 20 Cornwall Street, Woolloongabba QLD 4102, Australia.

\#These authors contributed equally

${ }^{*}$ Corresponding Author: Roland Stocker, $\mathrm{PhD}$

Vascular Biology Division, Victor Chang Cardiac Research Institute, Lowy Packer Building 450 Liverpool Road, Darlinghurst NSW 2010, Australia

Phone: +61292958712

\section{Supporting Information}

\section{Table of Contents}

Experimental Section

Supplementary Figures

Supplementary Tables

Supplementary References 


\section{Experimental Section}

\section{Materials}

For PCT lysis and digestion in a Barocycler (Pressure BioSciences, Inc.), all reagents were obtained from Sigma except for triethylammonium bicarbonate and the Rapid Trypsin/LysC digestion kit, which were purchased from Thermo Fisher Scientific and Promega, respectively. For metabolite extraction 3-azido-3'-deoxythymidine, dichloromethane and butylated hydroxytoluene were obtained from Sigma, LC-MS-grade acetonitrile and methanol were obtained from Merck and SPLASH Lipidomix was obtained from Avanti Polar Lipids. For LC-MS analysis all solvents and formic acid were of LC-MS grade obtained from Merck; ammonium hydroxide, ammonium formate and ammonium acetate were obtained from Sigma.

\section{Collection of Tissue Samples}

Male C57BL/6J WT and Apoe $e^{-/}$mice on a C57BL/6J background were obtained from BioCore (Victor Chang Cardiac Research Institute, Darlinghurst, Australia). C57BL/6J WT mice were fed a standard chow (Gordon's Specialty Stock Feeds, Yanderra, Australia) for 13 weeks. Apoe ${ }^{-/-}$mice (6 weeks of age) were fed Western Diet containing $22 \%$ fat and $0.15 \%$ cholesterol (SF00-219, Specialty Feeds, Western Australia) for 13 weeks. This Western Diet is formulated in accordance with the specifications of the Harlan Teklad diet TD88137. ${ }^{1}$ At the end of the intervention, mice were anesthetized with $4 \%$ isoflurane and sacrificed by exsanguination whilst maintained on $2 \%$ isoflurane. Blood was collected by cardiac puncture into a heparin-coated $1 \mathrm{~mL}$ syringe using a 25-gauge needle, and the animal then perfused with phosphate-buffered saline administered under physiological pressure. The hearts were cleared of surrounding adipose and connective tissue before being excised. Aortic roots were excised from the hearts, snap frozen on dry ice and stored at $-80{ }^{\circ} \mathrm{C}$. Aortic roots collected from C57BL/6J and Apoe $e^{-/}$mice constituted the lesion-free and lesion-containing tissue, respectively. The Animal Ethics Committees of the Garvan Institute of Medical Research/St Vincent's Hospital and the University of New South Wales approved all experiments.

\section{Effect of Drying Method on Lipid Oxidation in Organic Extracts}

Thoracic aortas from $3 \mathrm{Apoe}^{-/}$mice were lysed according to the protocol described in the main text. After tissue solubilization, whole lysate was resuspended in $100 \mu \mathrm{L}$ methanol/water $(3: 1, \mathrm{v} / \mathrm{v})$ and centrifuged at $13,000 \mathrm{~g}$ for $20 \mathrm{~min}$ at $4{ }^{\circ} \mathrm{C}$. The resulting pellet was reconstituted in $250 \mu \mathrm{L}$ dichloromethane/methanol (3:1, v/v) and mixed with $1 \mu \mathrm{L}$ of SPLASH Lipidomix and $100 \mu \mathrm{L} 1 \mathrm{mM}$ butylated hydroxytoluene. Samples were centrifuged 
at $10,000 \mathrm{~g}$ for $10 \mathrm{~min}$ at $4{ }^{\circ} \mathrm{C}$. The supernate was collected, split in half and transferred to glass LC-MS insert. The first aliquot was dried in the fume hood overnight and the second aliquot was dried under the flow of nitrogen gas (within about $5 \mathrm{~min}$ ). Dried extracts were reconstituted in $10 \mu \mathrm{L}$ methanol/toluene $(9: 1, \mathrm{v} / \mathrm{v})$ per $1 \mathrm{mg}$ tissue weight, centrifuged at $2,000 \mathrm{~g}$ for $5 \mathrm{~min}$ at $4{ }^{\circ} \mathrm{C}$ and transferred to LC-MS vials for analysis.

Lipid analysis was conducted using an Agilent 1290 Infinity II UHPLC system connected to a SCIEX TripleTOF 6600 mass spectrometer with a DuoSpray ${ }^{\mathrm{TM}}$ ion source. Lipid extracts were subjected to a Waters Acquity CSH C18 column $(2.1$ x $100 \mathrm{~mm}, 1.7 \mu \mathrm{m}$ particle size) eluted using a gradient comprised of mobile phase A (6:4 acetonitrile/water $(\mathrm{v} / \mathrm{v})$ with the addition of $10 \mathrm{mM}$ ammonium formate, and mobile phase $\mathrm{B}$ (9:1 isopropanol/acetonitrile (v/v) with the addition of $10 \mathrm{mM}$ ammonium formate. The flow rate was $0.3 \mathrm{~mL} / \mathrm{min}$ and the gradient consisted of $0-40 \%$ mobile phase $\mathrm{B}$ for $6 \mathrm{~min}$, increased to $100 \%$ in the following $24 \mathrm{~min}$, then held at $100 \% \mathrm{~B}$ for further $4 \mathrm{~min}$, before finally returning $0 \% \mathrm{~B}$ in $2 \mathrm{~min}$ and equilibrated for $4 \mathrm{~min}$. The column was maintained at $50{ }^{\circ} \mathrm{C}$, the autosampler at $4{ }^{\circ} \mathrm{C}$ and an injection volume of $5 \mu \mathrm{L}$ was used. The mass spectrometer parameters were as per 'Lipid Profiling of Organic Extracts'. The data files generated were processed by XCMS online, followed by data normalization to internal standards and biomass, log transformation and autoscaling.

As we adapted metabolomics and lipidomics workflow ${ }^{2}$, we anticipated that drying lipid extracts in the fume hood rather than under nitrogen gas could lead to ex vivo lipid oxidation. This oxidation could be the particularly relevant for bisallylic hydrogen-containing lipids (i.e., docosahexaenoic acid, DHA) decreasing the signal of these compounds. However, based on our control experiment we could not detect any features significantly decreased in abundance in lipid extracts dried in the fume hood (Figure S1), suggesting that the oxidized lipids detected in lesion-containing tissue were formed in vivo rather than ex vivo. Overlaid chromatograms of some DHA-containing lipid species showed no or little difference in the abundance of these molecules in response to the lipid extract drying method (Figures $\mathbf{S 2}$ and S3).

A Comparison of Metabolite and Feature Coverage from Mouse Arterial Segments Lysed Using Pressure Cycling Technology (PCT) Versus Bead Beating

The metabolite or feature count, peak area and number of metabolite/features with a coefficient of variation $(\mathrm{CV} \%)<25$ from data acquired using targeted and non-targeted metabolite and lipidomic analyses was compared between pressure cycling technology (PCT) 
and bead beating to lyse/homogenize arteries (Table S3). For each sample preparation $1 \mathrm{mg}$ of an atherosclerotic mouse arterial segment was used except for one bead beater procedure where $10 \mathrm{mg}$ of atherosclerotic tissue was used to assess the depth of metabolite output that may be lost using a smaller sample size. The number of replicates for each sample group was $\mathrm{n}=6-9$ except for the $10 \mathrm{mg}$ samples where $\mathrm{n}=3$.

\section{Targeted Metabolite Analysis}

Preparation of the $1 \mathrm{mg}$ arterial segments yielded similar metabolite coverage $( \pm 8 \%)$ and peak area $( \pm 24 \%)$ between pressure cycling technology (PCT) and bead beating to lyse/homogenize arteries (Table S3). For the $1 \mathrm{mg}$ arterial segments, CV used as an indication of precision and reproducibility, was 3-fold less for bead beater compared to PCT lysis. The metabolite count of $10 \mathrm{mg}$ tissue lysed using the bead-beater was only $20 \%$ higher than that obtained with $1 \mathrm{mg}$ tissue lysed using PCT or bead beater. However, the average peak area for metabolites was $\sim 4$-fold higher for the 10 compared with the $1 \mathrm{mg}$ tissue samples. CV (\%), was comparable between the PCT and $10 \mathrm{mg}$ bead beater samples.

\section{Non-Targeted Metabolomic Analysis}

Using non-targeted metabolomics and lipidomics analyses (both in positive and negative ion mode), the feature count was overall comparable between the four tissue sample preparations (Table S4). Average peak area was consistently higher for $10 \mathrm{mg}$ tissue compared with $1 \mathrm{mg}$ tissue (Table S4). 


\section{Supplementary Figures}

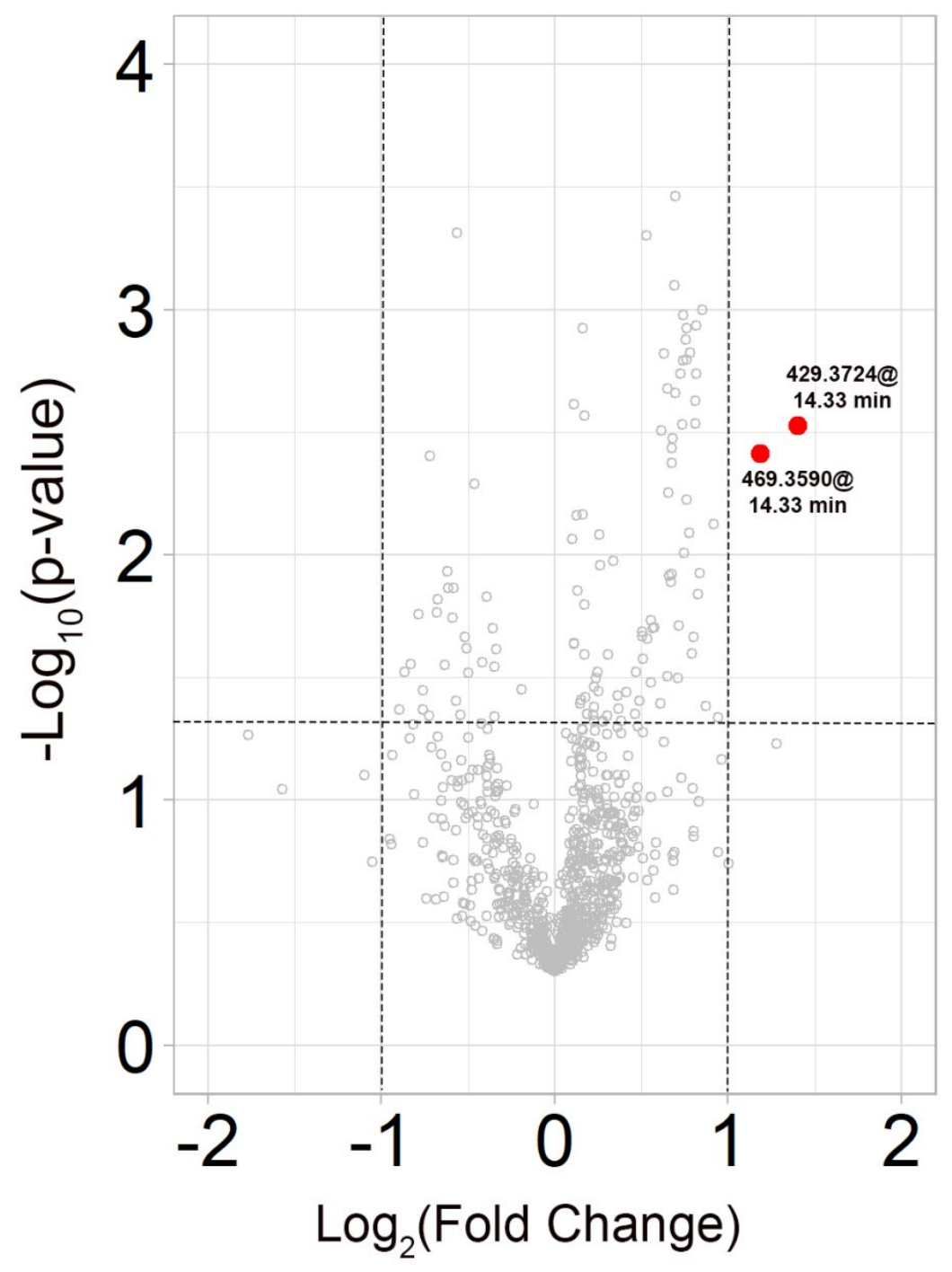

Figure S1. Volcano plot of lipid features identified in positive electrospray ionization mode. In red are the features with significantly higher abundance in lipid extracts dried in the fume hood overnight. Cut-off level is fold change $>2$, p-value $<0.05$ (FDR corrected). 
- XIC from Air_TA_204626_MS2_pos.wiff (sample 1) - Sample019, Experiment 1, +TOF MS (60 - 1500): 922.786 +/- 0.005 Da XXIC from Air_TA_204627_MS2_pos.wiff (sample 1) - Sample020, Experiment 1, +TOF MS $(60-1500): 922.786+1-0.005$ Da OXIC from Nitro TA 204626 MS2 pos. wiff (sample 1) - Sample009. Experiment 1, +TOF MS (60 - 1500): $922.786+1-0.005$ Da OXIC from Nitro_TA_204627_MS2_pos.wiff (sample 1) - Sample010, Experiment 1, +TOF MS $(60-1500): 922.786+1-0.005$ Da
OXIC from Nitro-TA_204819 MS2 pos.wiff (sample 1) - Sample008, Experiment 1, +TOF MS $(60-1500): 922.786+-0.005$ Da

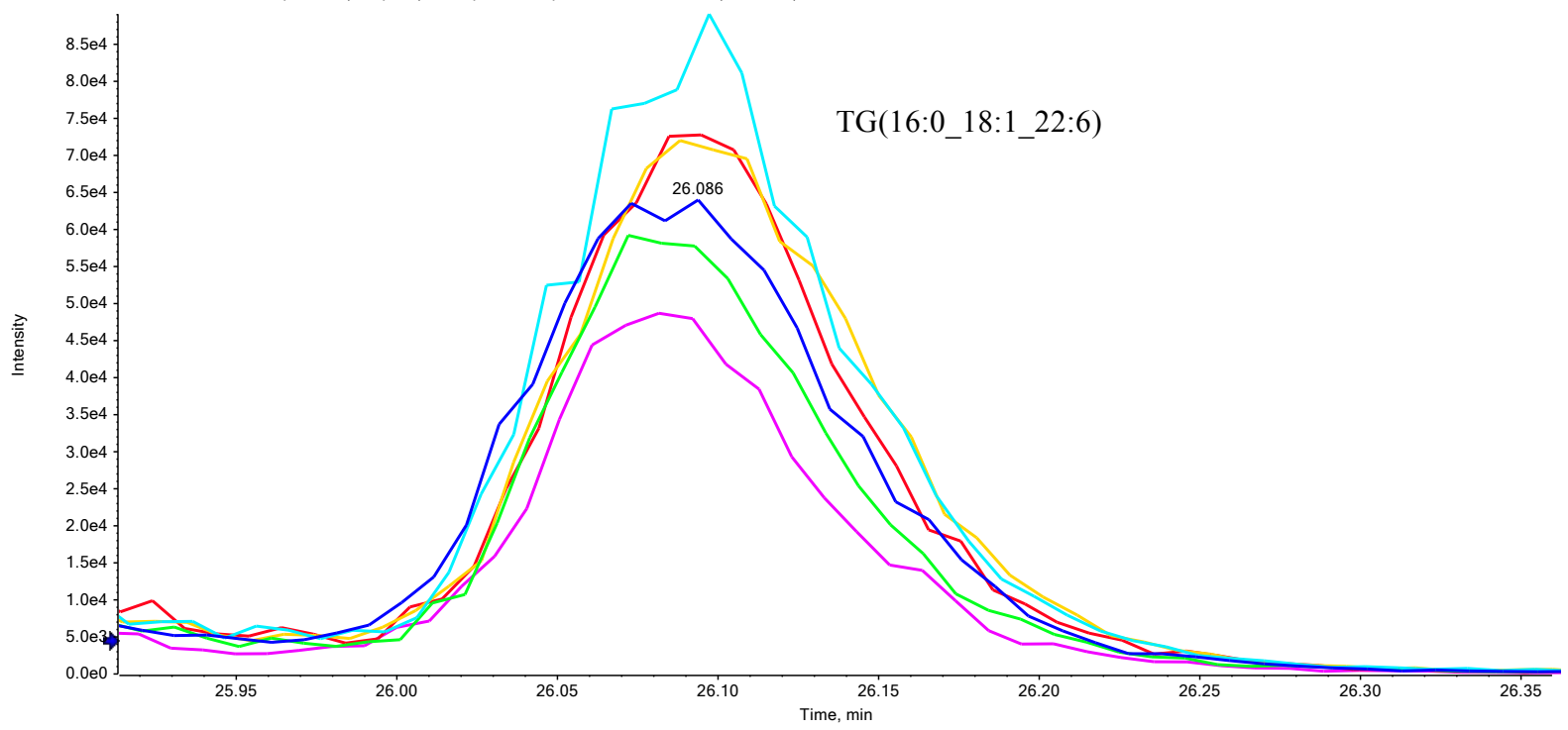

B

Spectrum from Air_TA_204626_MS2_pos.wiff (sample 1) - Sample019, Experiment 4, +TOF MS^2 (60 - 1500) from 26.048 min Precursor: $922.8 \mathrm{Da}$, CE: 35.0

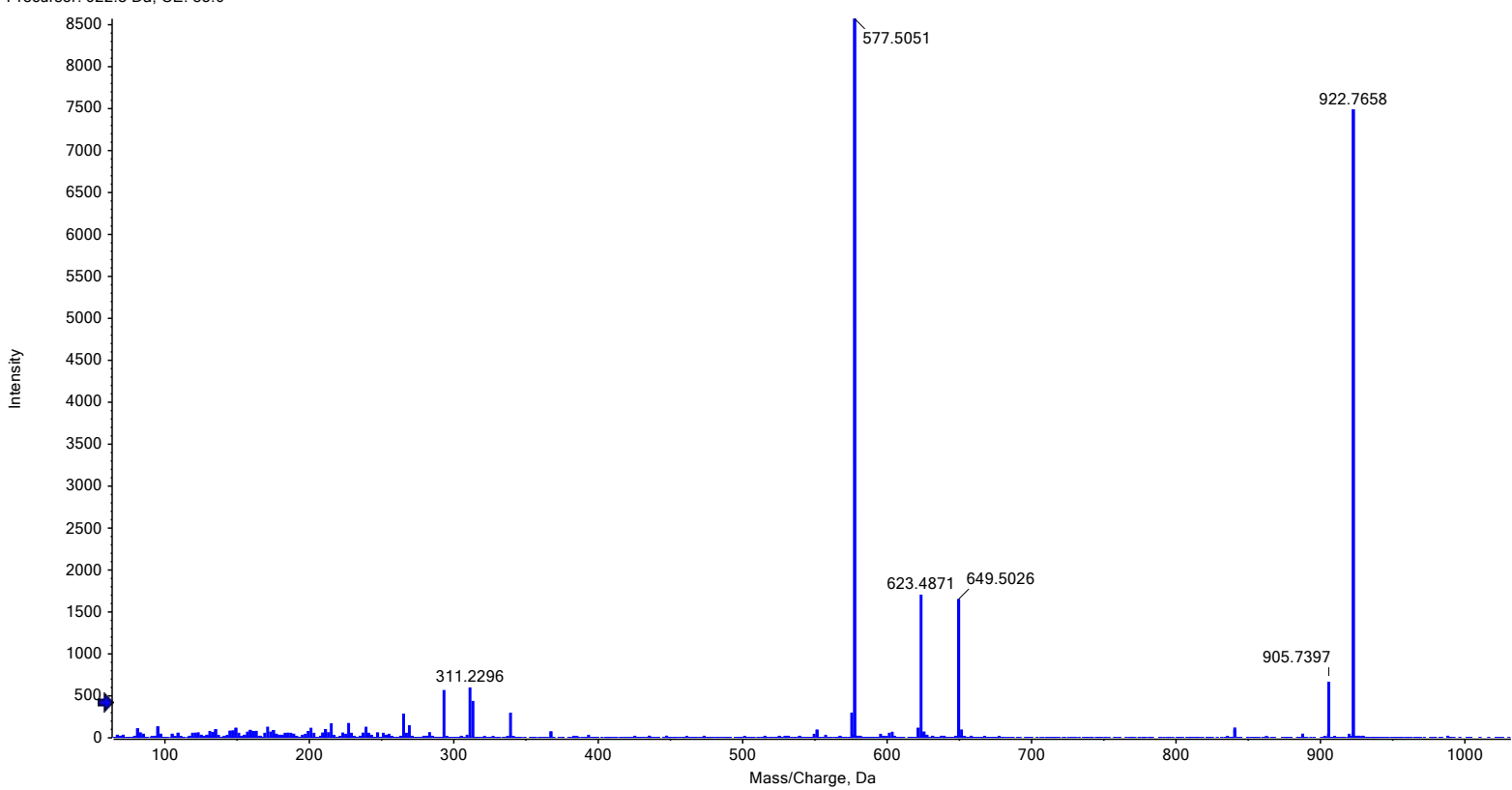

Figure S2. Electrospray ionization (positive ions) and tandem mass spectrometry of the triacylglyceride TAG(16:0_18:1_22:6). (A) Overlaid extracted ion chromatogram TAG(16:0_18:1_22:6). (B) Product ions are formed after collisional dissociation of the ammonium adduct $\left[\mathrm{M}+\mathrm{NH}_{4}\right]^{+}$at $\mathrm{m} / \mathrm{z}$ 922.8. Product ion $\mathrm{m} / \mathrm{z} 577.5$ is formed by loss of 22:6$\mathrm{RCOOH}+\mathrm{NH}_{3}(\mathrm{~m} / \mathrm{z} 345.3)$, product ion $\mathrm{m} / \mathrm{z} 623.5$ is formed by loss of $18: 1-\mathrm{RCOOH}+\mathrm{NH}_{3}$ $\left(\mathrm{m} / \mathrm{z}\right.$ 299.3), product ion $\mathrm{m} / \mathrm{z} 649.5$ is formed by loss of $16: 0-\mathrm{RCOOH}^{-\mathrm{NH}_{3}}(\mathrm{~m} / \mathrm{z} 273.3)$. 'Air_TA' refers to an organic extract dried in the fume hood, 'Nitro_TA' refers to an organic extract dried under nitrogen gas. 
- XIC from Air_TA_204626_MS2_pos.wiff (sample 1) - Sample019, Experiment 1, +TOF MS (60 - 1500): 950.817 +/- 0.005 Da

OXIC from Air-TA_204627_MS2_pos.wiff (sample 1) - Sample020, Experiment 1, +TOF MS $(60-1500): 950.817+$ +/- 0.005 Da

OXIC from Nitro_TA_204626_MS2_pos.wiff (sample 1) - Sample009, Experiment 1, +TOF MS $(60-1500): 950.817+1-0.005$ Da

OXIC from Nitro_TA_204627_MS2_pos.wiff (sample 1) - Sample010, Experiment 1, +TOF MS $(60-1500): 950.817+1-0.005$ Da
OXIC from Nitro_TA_204819_MS2_pos.wiff (sample 1) - Sample008, Experiment 1, +TOF MS $(60-1500): 950.817+-0.005$ Da

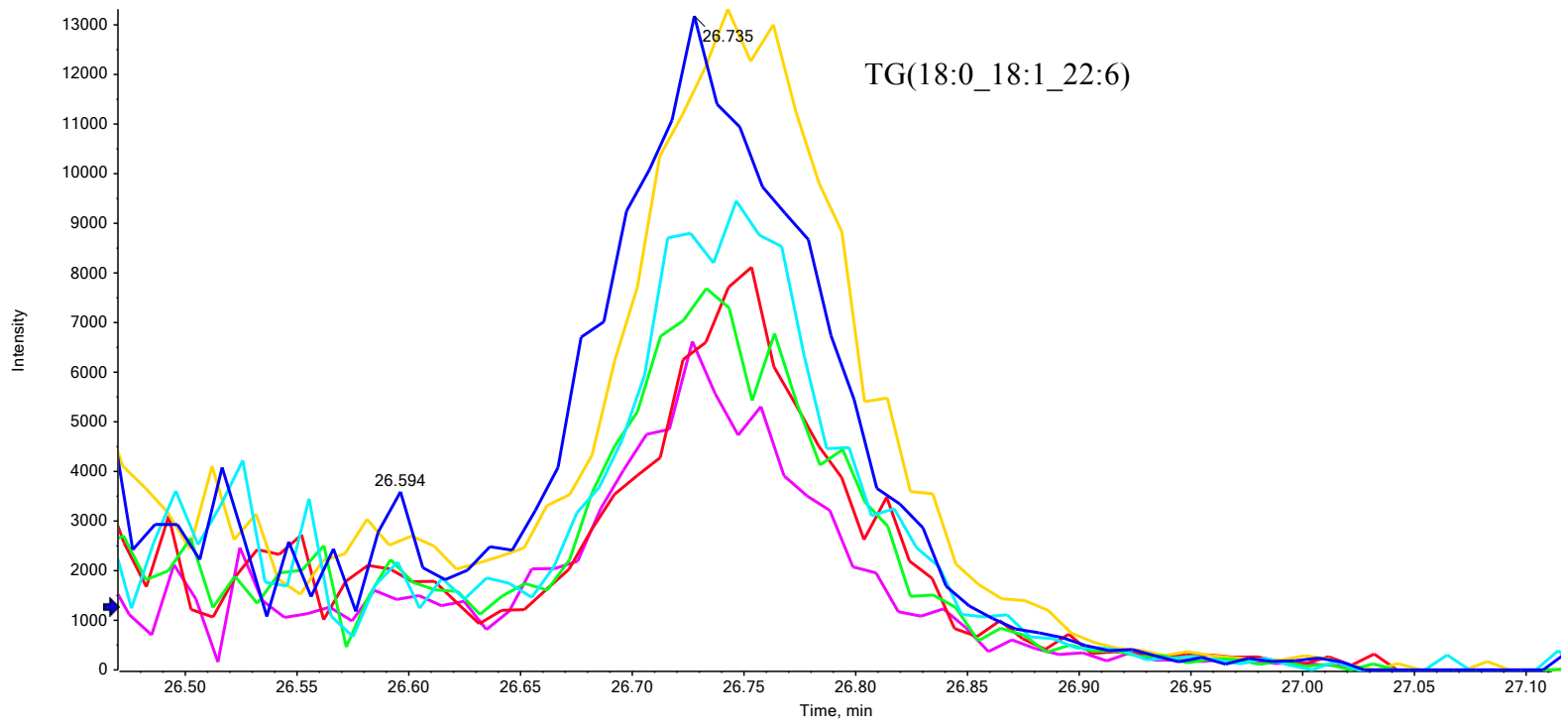

B

Spectrum from Air_TA_204626_MS2_pos.wiff (sample 1) - Sample019, Experiment 4, +TOF MS^2 (60 - 1500) from 26.693 min Precursor: $950.8 \mathrm{Da}$

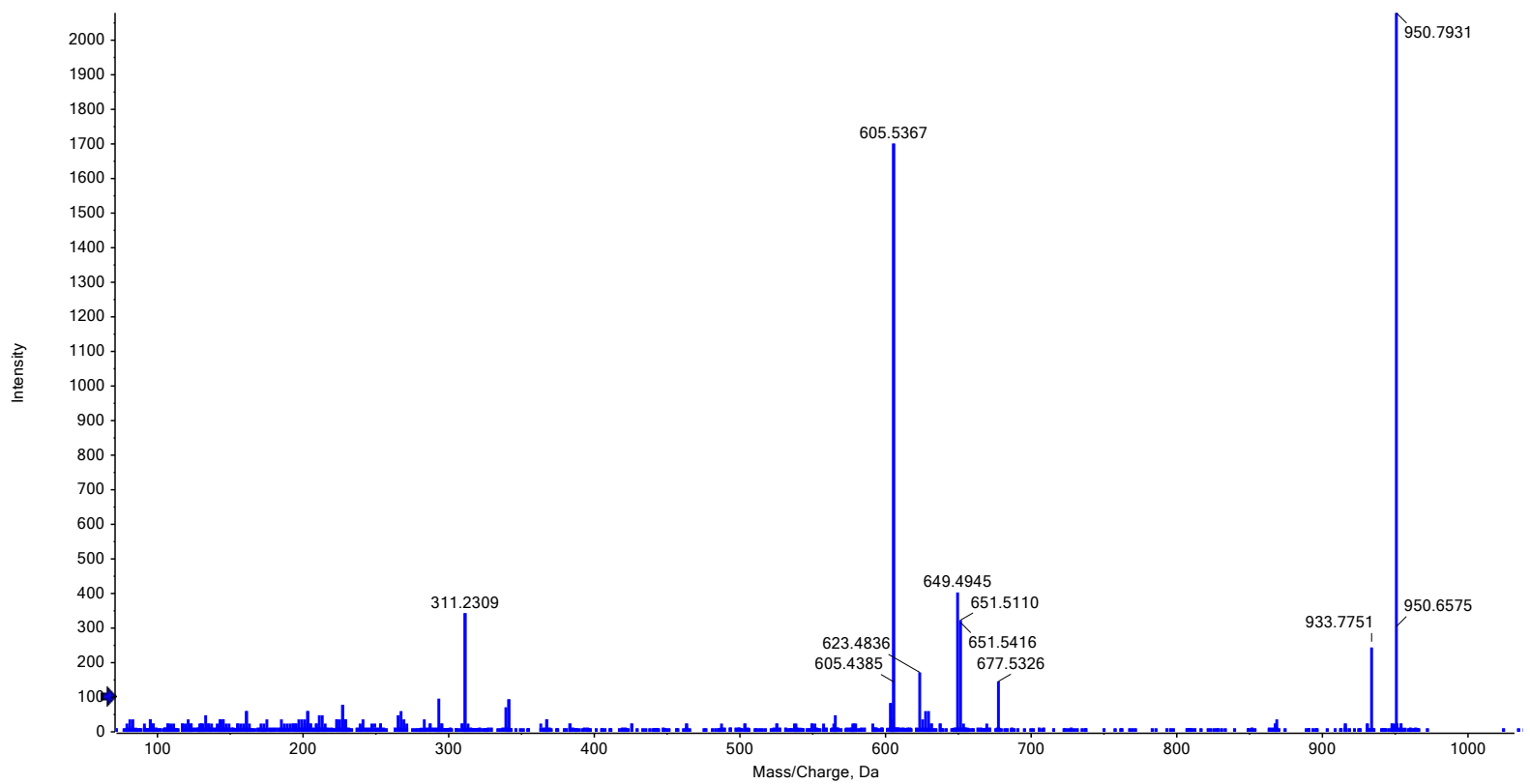

Figure S3. Electrospray ionization (positive ions) and tandem mass spectrometry of the triacylglyceride TAG(18:0_18:1_22:6). (A) Overlaid extracted ion chromatogram TAG(18:0_18:1_22:6). (B) Product ions are formed after collisional dissociation of the ammonium adduct $\left[\mathrm{M}+\mathrm{NH}_{4}\right]^{+}$at $\mathrm{m} / \mathrm{z}$ 950.8. Product ion $\mathrm{m} / \mathrm{z} 605.5$ is formed by loss of 22:6$\mathrm{RCOOH}+\mathrm{NH}_{3}(\mathrm{~m} / \mathrm{z} 345.3)$, product ion $\mathrm{m} / \mathrm{z} 651.5$ is formed by loss of $18: 1-\mathrm{RCOOH}+\mathrm{NH}_{3}$ $\left(\mathrm{m} / \mathrm{z}\right.$ 299.3), product ion $\mathrm{m} / \mathrm{z} 649.5$ is formed by loss of $18: 0-\mathrm{RCOOH}^{-\mathrm{NH}_{3}}(\mathrm{~m} / \mathrm{z} 301.3)$. 'Air_TA' refers to an organic extract dried in the fume hood, 'Nitro_TA' refers to an organic extract dried under nitrogen gas. 

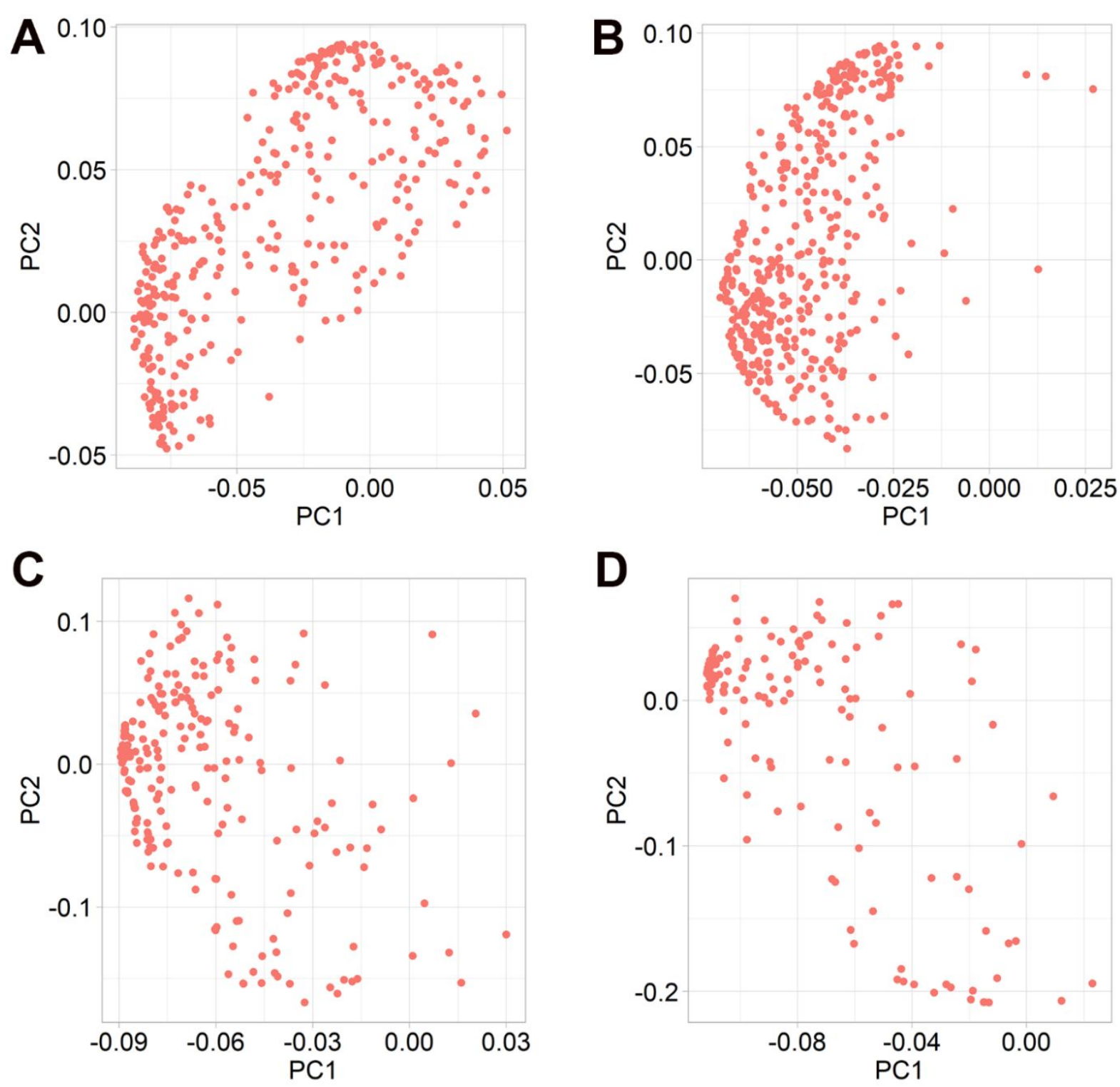

Figure S4. Loading plots of principal component analysis (PCA) generated using (A) lipid features (organic extract) identified in positive electrospray ionization mode and (B) in negative electrospray ionization mode; loading plots of principal component analysis (PCA) generated using $(\mathrm{C})$ metabolite features (aqueous extract) identified in positive electrospray ionization mode and (D) in negative electrospray ionization mode. 


\section{Supplementary Tables}

Table S1: Metabolites detected using targeted metabolite analysis that are significantly increased in lesion tissue

\begin{tabular}{|c|c|c|c|c|}
\hline Pathway & Metabolite & $\begin{array}{c}\text { Fold } \\
\text { Change }\end{array}$ & P-value & $\begin{array}{c}\text { Reported } \\
\text { association } \\
\text { with CAD }\end{array}$ \\
\hline \multirow{6}{*}{$\begin{array}{l}\text { Amino acid } \\
\text { metabolism }\end{array}$} & L-Phenylalanine & 4.0 & $<0.0001$ & 3 \\
\hline & L-Serine & 2.3 & $<0.0001$ & 3-5 \\
\hline & L-Tyrosine & 2.2 & 0.0002 & 3-5 \\
\hline & L-Tryptophan & 1.9 & 0.0002 & 3-5 \\
\hline & L-Methionine & 1.8 & $<0.0001$ & 3,4 \\
\hline & L-Glutamine & 1.5 & 0.0158 & $6-10$ \\
\hline \multicolumn{5}{|l|}{ Energy Metabolism } \\
\hline \multirow{3}{*}{$\begin{array}{l}\text { Coenzyme A } \\
\text { biosynthesis } \\
\text { Glycolysis }\end{array}$} & D-Pantothenic acid & 4.9 & $<0.0001$ & \\
\hline & $\begin{array}{l}\text { Dihydroxyacetone } \\
\text { phosphate }\end{array}$ & 3.0 & 0.0003 & 10 \\
\hline & Lactic acid & 1.5 & 0.0149 & 11 \\
\hline \multirow[t]{2}{*}{ TCA cycle } & L-Malic acid & 4.0 & 0.0008 & \\
\hline & Ketovaleric acid & 2.5 & 0.0015 & 12 \\
\hline \multirow{2}{*}{$\begin{array}{l}\text { Pentose phosphate } \\
\text { pathway }\end{array}$} & D-Ribose 5-phosphate & 2.3 & 0.0018 & 10,11 \\
\hline & $\begin{array}{l}\text { D-Sedoheptulose-7- } \\
\text { phosphate }\end{array}$ & 1.9 & 0.0021 & 10,11 \\
\hline $\begin{array}{l}\text { Branched chain } \\
\text { amino acid }\end{array}$ & L-Leucine & 1.9 & 0.0132 & $3-5,13$ \\
\hline ADP-ATP recycling & Creatine & 1.5 & 0.0167 & \\
\hline \multirow[t]{5}{*}{ Purine metabolism } & Xanthosine & 4.9 & $<0.0001$ & 5 \\
\hline & Adenine & 3.4 & 0.0031 & 5,11 \\
\hline & Xanthine & 2.5 & $<0.0001$ & 5,11 \\
\hline & Uric acid & 2.0 & 0.001 & 5,11 \\
\hline & Hypoxanthine & 2.0 & 0.0015 & 5,11 \\
\hline Immunometabolite & L-Hydroxyglutaric acid & 3.0 & 0.0194 & 11 \\
\hline $\begin{array}{l}\text { Pyrimidine } \\
\text { metabolism }\end{array}$ & Uracil & 2.6 & 0.0001 & 14 \\
\hline
\end{tabular}




\begin{tabular}{llccc} 
Urea cycle & L-Aspartic Acid & 2.0 & 0.0034 & 11 \\
$\begin{array}{l}\text { Cyanoamino acid } \\
\text { metabolism }\end{array}$ & L-Glutamic acid & 1.8 & 0.0003 & 9 \\
$\begin{array}{l}\text { Collagen synthesis } \\
\begin{array}{l}\text { Glycerophospholipid } \\
\text { metabolism }\end{array}\end{array}$ & L-Threonine & 1.7 & 0.001 & \\
$\begin{array}{l}\text { Primary bile acid } \\
\text { biosynthesis }\end{array}$ & Taurine & 1.7 & 0.0185 & 3 \\
$\begin{array}{l}\text { Cholesterol transport } \\
\text { D-Erythro- }\end{array}$ & 1.4 & 0.0103 & \\
\hline
\end{tabular}

Table S2: Metabolites detected using targeted metabolite analysis that are significantly decreased in lesion tissue

\begin{tabular}{llccc}
\hline \multicolumn{1}{c}{ Pathway } & Metabolite & $\begin{array}{c}\text { Fold } \\
\text { Change }\end{array}$ & P-value & $\begin{array}{c}\text { Reported } \\
\text { association } \\
\text { with CAD }\end{array}$ \\
\hline $\begin{array}{l}\text { Frutose and mannose } \\
\text { metabolism }\end{array}$ & L-Sorbose & 0.4 & 0.0031 & \\
$\begin{array}{l}\text { Galactose metabolism } \\
\text { Purine metabolism }\end{array}$ & myo-Inositol & 0.5 & 0.0014 & \\
& $\begin{array}{l}\text { D-Maltose } \\
\text { Guanosine }\end{array}$ & 0.6 & 0.013 \\
& $\begin{array}{l}\text { B-Nicotinamide } \\
\text { adenine } \\
\text { dinucleotide }\end{array}$ & 0.4 & $<0.0187$ \\
& & & \\
\hline
\end{tabular}

Table S3: Peak area, count and coefficient of variation ( $\mathrm{CV} \%$ ) of aqueous metabolites detected in mouse arterial lesions by targeted metabolite analysis

\begin{tabular}{lccc}
\hline & Average peak area & $\begin{array}{c}\text { Metabolite } \\
\text { count } \\
\text { Mean } \pm \text { SD }\end{array}$ & $\begin{array}{c}\text { Number of features with } \\
\text { CV \% }<25 *\end{array}$ \\
\hline Bead beater $(10 \mathrm{mg})$ & 237,564 & $105 \pm 1.5$ & 18 \\
Bead beater $(1 \mathrm{mg})$ & 76,941 & $89 \pm 10.4$ & 6 \\
Barocycler & 68,368 & $87 \pm 2.4$ & 21 \\
metabolomics only & 58,120 & 19 \\
Barocycler multi-omics & *Coefficient of variation was determined by dividing the standard deviation by the mean of \\
the peak area for each metabolite detected. Comparable results were obtained using a non- \\
targeted metabolomics analysis (data not shown).
\end{tabular}


Table S4: Peak area, count and coefficient of variation ( $\mathrm{CV} \%$ ) of metabolites detected in mouse arterial lesions by non-targeted approach

\begin{tabular}{|c|c|c|c|}
\hline & Average peak area & $\begin{array}{c}\text { Feature } \\
\text { count }\end{array}$ & $\begin{array}{c}\text { Number of features with } \\
\text { CV } \%<25 *\end{array}$ \\
\hline \multicolumn{4}{|c|}{ Metabolomics - positive mode } \\
\hline Bead beater $(10 \mathrm{mg})$ & 195,392 & 4,518 & 1,103 \\
\hline Bead beater (1 mg) & 88,495 & 3,697 & 111 \\
\hline $\begin{array}{l}\text { Barocycler } \\
\text { metabolomics only }\end{array}$ & 59,828 & 3,533 & 523 \\
\hline Barocycler multi-omics & 69,614 & 3,602 & 891 \\
\hline \multicolumn{4}{|c|}{ Metabolomics - negative mode } \\
\hline Bead beater $(10 \mathrm{mg})$ & 209,629 & 4,560 & 775 \\
\hline Bead beater $(1 \mathrm{mg})$ & 169,633 & 4,262 & 639 \\
\hline $\begin{array}{l}\text { Barocycler } \\
\text { metabolomics only }\end{array}$ & 56,041 & 4,386 & 536 \\
\hline Barocycler multi-omics & 57,772 & 4,307 & 1,345 \\
\hline \multicolumn{4}{|c|}{ Lipidomics - positive mode } \\
\hline Bead beater $(10 \mathrm{mg})$ & $7,907,955$ & 3,860 & 2,221 \\
\hline Bead beater $(1 \mathrm{mg})$ & $3,007,735$ & 3,684 & 408 \\
\hline $\begin{array}{l}\text { Barocycler } \\
\text { metabolomics only }\end{array}$ & $4,637,279$ & 3,537 & 611 \\
\hline Barocycler multi-omics & $2,923,944$ & 3,605 & 1,432 \\
\hline \multicolumn{4}{|c|}{ Lipidomics - negative mode } \\
\hline Bead beater $(10 \mathrm{mg})$ & $2,498,650$ & 4,276 & 1,392 \\
\hline Bead beater (1 mg) & $1,167,179$ & 3,919 & 428 \\
\hline $\begin{array}{l}\text { Barocycler } \\
\text { metabolomics only }\end{array}$ & $1,746,770$ & 3,962 & 380 \\
\hline Barocycler multi-omics & $1,471,587$ & 4,084 & 1,154 \\
\hline
\end{tabular}




\section{References}

(1) Letters, J. M.; Witting P. K.; Christison J. K.; Westin Eriksson A.; Pettersson K.; Stocker R. Time-dependent changes to lipids and antioxidants in plasma and aortas of apolipoprotein E knockout mice. J. Lipid Res. 1999, 40, 1104-1112.

(2) Vorkas, P. A.; Isaac G.; Anwar M. A.; Davies A. H.; Want E. J.; Nicholson J. K.; Holmes E. Untargeted UPLC-MS profiling pipeline to expand tissue metabolome coverage: application to cardiovascular disease. Anal Chem. 2015, 87, 4184-93.

(3) Zhang, L.; Wei T. T.; Li Y.; Li J.; Fan Y.; Huang F. Q.; Cai Y. Y.; Ma G.; Liu J. F.; Chen Q. Q.; Wang S. L.; Li H.; Alolga R. N.; Liu B.; Zhao D. S.; Shen J. H.; Wang X. M.; Zhu W.; Li P.; Qi L. W. Functional metabolomics characterizes a key role for $\mathrm{N}$ acetylneuraminic acid in coronary artery diseases. Circulation. 2018, 137, 1374-1390.

(4) Shah, S. H.; Bain J. R.; Muehlbauer M. J.; Stevens R. D.; Crosslin D. R.; Haynes C.; Dungan J.; Newby L. K.; Hauser E. R.; Ginsburg G. S.; Newgard C. B.; Kraus W. E. Association of a peripheral blood metabolic profile with coronary artery disease and risk of subsequent cardiovascular events. Circ. Cardiovasc. Genet. 2010, 3, 207-214.

(5) Jung, S.; Song S. W.; Lee S.; Kim S. H.; Ann S. J.; Cheon E. J.; Yi G.; Choi E. Y.; Lee S. H.; Joo H. C.; Ryu D. H.; Lee S. H.; Hwang G. S. Metabolic phenotyping of human atherosclerotic plaques: Metabolic alterations and their biological relevance in plaquecontaining aorta. Atherosclerosis. 2018, 269, 21-28.

(6) Tomas, L.; Edsfeldt A.; Mollet I. G.; Perisic Matic L.; Prehn C.; Adamski J.; PaulssonBerne G.; Hedin U.; Nilsson J.; Bengtsson E.; Goncalves I.; Björkbacka H. Altered metabolism distinguishes high-risk from stable carotid atherosclerotic plaques. Eur. Heart J. 2018, 39, 2301-2310.

(7) Dang, V. T.; Huang A.; Zhong L. H.; Shi Y.; Werstuck G. H. Comprehensive plasma metabolomic analyses of atherosclerotic progression reveal alterations in glycerophospholipid and sphingolipid metabolism in apolipoprotein E-deficient mice. Sci. Rep. 2016, 6, 35037.

(8) Shah, S. H.; Hauser E. R.; Bain J. R.; Muehlbauer M. J.; Haynes C.; Stevens R. D.; Wenner B. R.; Dowdy Z. E.; Granger C. B.; Ginsburg G. S.; Newgard C. B.; Kraus W. E. High heritability of metabolomic profiles in families burdened with premature cardiovascular disease. Mol. Syst. Biol. 2009, 5, 258.

(9) Mudge, G. H., Jr.; Mills R. M., Jr.; Taegtmeyer H.; Gorlin R.; Lesch M. Alterations of myocardial amino acid metabolism in chronic ischemic heart disease. J. Clin. Invest. 1976, 58, 1185-1192.

(10) Yamashita, A.; Zhao Y.; Matsuura Y.; Yamasaki K.; Moriguchi-Goto S.; Sugita C.; Iwakiri T.; Okuyama N.; Koshimoto C.; Kawai K.; Tamaki N.; Zhao S.; Kuge Y.; Asada Y. Increased metabolite levels of glycolysis and pentose phosphate pathway in rabbit atherosclerotic arteries and hypoxic macrophage. PloS one. 2014, 9, e86426.

(11) Matsuura, Y.; Yamashita A.; Zhao Y.; Iwakiri T.; Yamasaki K.; Sugita C.; Koshimoto C.; Kitamura K.; Kawai K.; Tamaki N.; Zhao S.; Kuge Y.; Asada Y. Altered glucose metabolism and hypoxic response in alloxan-induced diabetic atherosclerosis in rabbits. PloS one. 2017, 12, e0175976.

(12) Sun, H.; Olson K. C.; Gao C.; Prosdocimo D. A.; Zhou M.; Wang Z.; Jeyaraj D.; Youn J. Y.; Ren S.; Liu Y.; Rau C. D.; Shah S.; Ilkayeva O.; Gui W. J.; William N. S.; Wynn R. M.; Newgard C. B.; Cai H.; Xiao X.; Chuang D. T.; Schulze P. C.; Lynch C.; Jain M. K.; Wang Y. Catabolic defect of branched-chain amino acids promotes heart failure. Circulation. 2016, 133, 2038-2049.

(13) Yang, R. Y.; Wang S. M.; Sun L.; Liu J. M.; Li H. X.; Sui X. F.; Wang M.; Xiu H. L.; Wang S.; He Q.; Dong J.; Chen W. X. Association of branched-chain amino acids 
with coronary artery disease: A matched-pair case-control study. Nutr. Metab. Cardiovasc. Dis. 2015, 25, 937-942.

(14) Vorkas, P. A.; Shalhoub J.; Isaac G.; Want E. J.; Nicholson J. K.; Holmes E.; Davies A. H. Metabolic phenotyping of atherosclerotic plaques reveals latent associations between free cholesterol and ceramide metabolism in atherogenesis. J. Proteome Res. 2015, 14, 1389-1399. 